\title{
E-SiCure Collaboration Project: Silicon Carbide Material Studies and Detector Prototype Testing at the JSI TRIGA Reactor
}

\author{
Vladimir Radulović \\ Jožef Stefan Institute \\ Ljubljana, Slovenia \\ vladimir.radulovic@ijs.si
}

\author{
Klemen Ambrožič \\ Jožef Stefan Institute \\ Ljubljana, Slovenia
}

klemen.ambrozic@ijs.si

\author{
Luka Snoj \\ Jožef Stefan Institute \\ Ljubljana, Slovenia
}

luka.snoj@ijs.si

\author{
Ivana Capan \\ Rudjer Bošković Institute \\ Zagreb, Croatia \\ ivana.capan@irb.hr
}

\author{
Adam Sarbutt \\ ANSTO \\ Lucas Heights, Australia \\ ars@ansto.gov.au \\ José Coutinho \\ University of Aveiro \\ Aveiro, Portugal \\ jose.coutinho@ua.pt
}

\author{
Tomislav Brodar \\ Rudjer Bošković Institute \\ Zagreb, Croatia \\ tomislav.brodar@irb.hr
}

\author{
Yuichi Yamazaki \\ QST \\ Takasaki Gunma, Japan \\ yamazaki.yuichi@qst.go.jp
}

\author{
Željko Pastuović \\ uucas Heights, Australia \\ zkp@ansto.gov.au
}

\author{
Takeshi Ohshima \\ QST \\ Takasaki Gunma, Japan \\ ohshima.takeshi@qst.go.jp
}

\begin{abstract}
In 2016, the "E-SiCure" project (standing for Engineering Silicon Carbide for Border and Port Security), funded by the NATO Science for Peace and Security Programme, was launched. The main objective is to combine theoretical, experimental and applied research towards the development of radiation-hard $\mathrm{SiC}$-based detectors of special nuclear materials (SNM), and by that way, to enhance border and port security barriers. Along the plan, material modification processes are employed firstly to study, and secondly to manipulate the most severe electrically active defects (which trap or annihilate free charge carriers), by specific ion implantation and defect engineering. This paper gives an overview of the experimental activities performed at the JSI TRIGA reactor in the framework of the E-SiCure project. Initial activities were aimed at obtaining information on the radiation hardness of $\mathrm{SiC}$ and at the study of the energy levels of the defects induced by neutron irradiation. Several Schottky barrier diodes were fabricated out of nitrogen-doped epitaxial grown $4 \mathrm{H}-\mathrm{SiC}$, and irradiated under Cd filters in the PT irradiation channel in the JSI TRIGA reactor with varying neutron fluence levels. Neutron-induced defects in the material were studied using temperature dependent current-voltage (I-V), capacitance-voltage (C-V) and Deep-Level Transient Spectroscopy (DLTS) measurements. Our prototype neutron detectors are configured as $\mathbf{4 H}-\mathrm{SiC}$-based Schottky barrier diodes for detection of secondary charged particles (tritons, alphas and lithium atoms) which are result of thermal neutron conversion process in ${ }^{10} \mathrm{~B}$ and ${ }^{6} \mathrm{LiF}$ layers above the surface of the $4 \mathrm{H}-\mathrm{SiC}$ diodes. For field testing of neutron detectors using a broad beam of reactor neutrons we designed a standalone prototype detection system consisting of a preamplifier, shaping amplifier and a multichannel analyser operated by a laptop computer. The reverse bias for the detector diode and the power to electronic system are provided by a standalone battery-powered voltage source. The detector functionality was
\end{abstract}

NATO Science for Peace and Security Programme established through measurements using an ${ }^{241} \mathrm{Am}$ alpha particle source. Two dedicated experimental campaigns were performed at the JSI TRIGA reactor. The registered pulse height spectra from the detectors, using both ${ }^{10} \mathrm{~B}$ and ${ }^{6} \mathrm{LiF}$ neutron converting layers, clearly demonstrated the neutron detection abilities of the $\mathrm{SiC}$ detector prototypes.

Keywords-Silicon carbide, Neutron detection, Neutron converter, JSI TRIGA reactor

\section{INTRODUCTION}

Increasingly complex risks like geopolitical instability or decentralized terrorism threats, have led to the urge for deploying nuclear screening systems for detection of illicit trafficking of nuclear materials, and from that, to a growing interest in the field of research and development of new radiation detection technologies suitable for homeland security applications. Recent progress in the manufacturing of highquality bulk and epitaxial silicon carbide $(\mathrm{SiC})$ and processing technologies for fabrication of $\mathrm{SiC}$-based electronic devices, could enable unprecedented opportunities for future SiC-based detection of neutron and alpha particle emissions. Unlike existing and commonly used gas-based neutron detectors, SiC-based devices have the potential to be simultaneously portable, operable at room temperature and radiation hard. The main objective of the E-SiCure project is to combine theoretical, experimental and applied research towards the development of radiation-hard SiC-based detectors of special nuclear materials (SNM), and therefore to enhance border and port security barriers, which is achieved through studies of material radiation hardness and modification processes in order to manipulate the most severe electrically active defects 
which trap or annihilate free charge carriers, by specific ion implantation and defect engineering. This paper presents the experimental activities performed at the JSI TRIGA reactor in the framework of the E-SiCure project. Section 2 presents the study of radiation-induced defects in neutron irradiated SiC diodes with Deep-Level Transient Spectroscopy. Section 3 presents the realization of prototype $\mathrm{SiC}$ detectors and initial functional testing with a ${ }^{241} \mathrm{Am}$ radiation source. Section 4 presents a dedicated experimental campaign performed at the JSI TRIGA reactor aimed at demonstrating the neutron detection capability of SiC-based detector prototypes.

\section{Neutron-Induced Defects in SiC}

To study neutron induced defects in SiC, n-type silicon carbide Schottky barrier diodes (SBDs) were fabricated onto nitrogen-doped epitaxial grown $4 \mathrm{H}-\mathrm{SiC}$ single crystal layers approximately $25 \mu \mathrm{m}$ thick, their lateral dimensions being $1 \mathrm{~mm}$ by $1 \mathrm{~mm}$ [1]. The Schottky diodes were irradiated either upon bare exposure or inside $\mathrm{Cd}$ thermal neutron filters with a wall thickness of $1 \mathrm{~mm}$ in the $250 \mathrm{~kW}$ JSI TRIGA reactor in Ljubljana, Slovenia. The irradiations were performed in the Pneumatic Tube (PT) irradiation facility, located in the F24 position in the outermost $\mathrm{F}$ ring of the reactor; the neutron fluences ranged from $10^{8} \mathrm{n} \mathrm{cm}^{-2}$ to $10^{15} \mathrm{n}$ $\mathrm{cm}^{-2}$. The neutron spectrum in the PT irradiation channel was obtained from Monte Carlo neutron transport calculations with the MCNP6 code [2] in conjunction with the ENDF/B-VII.1 nuclear data library [3]. A verified and validated computational model of the JSI TRIGA reactor was employed, based on the JSI TRIGA criticality benchmark model [4], featured in the International Handbook of Evaluated Critical Safety Benchmark Experiments [5]. The computational model is in permanent use at the Reactor Physics Department of the JSI for research purposes (computer code and nuclear data validation) and in support of experimental campaigns performed at the reactor [6][7][8]. Over time, the model has been considerably updated and refined. It has been validated against experimental measurements for calculations of the multiplication factor $k_{e f f}$ [4], reactor kinetic parameters [9], neutron flux and reaction rate distributions [10][11][12]. The neutron spectrum obtained from Monte Carlo calculations underwent a subsequent process of neutron spectrum adjustment to measured reaction rate ratios. The adjustment process was performed using the JSIdeveloped GRUPINT code package [13]. Fig. 1 displays the adjusted bare and Cd-filtered neutron spectrum in the PT facility.

A total of 8 samples were irradiated at different reactor power levels $(2.5 \mathrm{~W}, 25 \mathrm{~W}$, and $250 \mathrm{~kW})$, specifying different irradiation times, in order to span a range of 7 orders of magnitude in the neutron fluence delivered to the samples $\left(10^{8}\right.$ $\mathrm{n} \mathrm{cm}-2$ to $10^{15} \mathrm{n} \mathrm{cm}^{-2}$ nominal fluence range). Activation measurements for the ${ }^{197} \mathrm{Au}(n, \gamma)$ reaction were performed for each power level used for the irradiations in order to deduce the total neutron flux. The subcadmium flux was obtained from the characterized neutron spectrum and the Cd cutoff energy for the thickness of $1 \mathrm{~mm}$, i.e. $0.55 \mathrm{eV}$. Table 1 reports

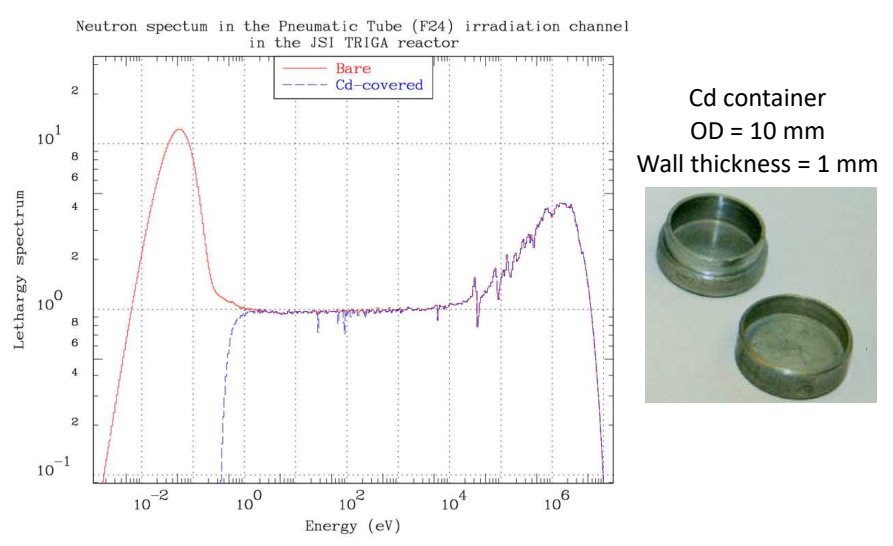

Fig. 1. Neutron spectra in the Pneumatic Tube (PT) irradiation channel, located in the F24 position in the core of the JSI TRIGA reactor. Red: bare spectrum. Blue: spectrum under $\mathrm{Cd}$ cover.

details of the neutron irradiation conditions for the selected samples. The calculated uncertainties in the neutron fluences were obtained from uncertainties in the neutron flux and the irradiation time.

TABLE I

IRRADIATION DETAILS, SUBCADMIUM NEUTRON FLUX LEVELS AND 1- $\sigma$ EXPERIMENTAL UNCERTAINTIES, SAMPLE NEUTRON FLUENCE VALUES AND $1-\sigma$ EXPERIMENTAL UNCERTAINTIES.

\begin{tabular}{|c|c|c|c|c|}
\hline ID & $\begin{array}{c}\text { Irr. } \\
\text { time }[\mathrm{s}]\end{array}$ & $\begin{array}{c}\text { P } \\
{[\mathrm{W}]}\end{array}$ & $\begin{array}{c}\text { Sub-Cd flux } \\
{\left[\mathrm{n} \mathrm{cm}^{-2} \mathrm{~s}^{-1}\right]}\end{array}$ & $\begin{array}{c}\text { Fluence } \\
{\left[\mathrm{n} \mathrm{cm}^{-2}\right]}\end{array}$ \\
\hline R6 & 3.0 & 2.5 & $3.66 \times 10^{7} \pm 2.7 \%$ & $1.10 \times 10^{8} \pm 9.8 \%$ \\
R10 & 30 & 2.5 & $3.66 \times 10^{7} \pm 2.7 \%$ & $1.10 \times 10^{9} \pm 2.9 \%$ \\
R11 & 300 & 2.5 & $3.66 \times 10^{7} \pm 2.7 \%$ & $1.10 \times 10^{10} \pm 2.7 \%$ \\
R9 & 273 & 25 & $3.44 \times 10^{8} \pm 2.9 \%$ & $9.39 \times 10^{10} \pm 2.9 \%$ \\
R12 & 2730 & 25 & $3.44 \times 10^{8} \pm 2.9 \%$ & $9.39 \times 10^{11} \pm 2.9 \%$ \\
1E13 & 2.73 & $250 \mathrm{k}$ & $3.66 \times 10^{12} \pm 2.7 \%$ & $1.00 \times 10^{13} \pm 10.7 \%$ \\
IE14 & 27.3 & $250 \mathrm{k}$ & $3.66 \times 10^{12} \pm 2.7 \%$ & $1.00 \times 10^{14} \pm 2.9 \%$ \\
1E15 & 273 & $250 \mathrm{k}$ & $3.66 \times 10^{12} \pm 2.7 \%$ & $1.00 \times 10^{15} \pm 2.7 \%$ \\
\hline
\end{tabular}

Neutron induced defects in the samples were studied using temperature dependent current-voltage (I-V), capacitancevoltage (C-V) and Deep-Level Transient Spectroscopy (DLTS) measurements. I-V and $\mathrm{C}-\mathrm{V}$ measurements were carried out using a Keithley 6487 Picoammeter/Voltage Source and a Keithley 4200 Semiconductor characterization system. DLTS and Laplace-DLTS measurements were carried out using a Boonton 7200 capacitance meter, a NI PCI-6251 DAQ and Laplace DLTS software. DLTS and Laplace-DLTS measurements were performed in the temperature range from $100 \mathrm{~K}$ up to $380 \mathrm{~K}$. Temperature dependent I-V measurements revealed that the fast neutron irradiation did not affect the transport properties of SBDs for neutron fluences lower than $10^{12} \mathrm{n}$ $\mathrm{cm}^{-2}$. An increase in the series resistance and decrease of the capacitance of neutron irradiated samples was observed first after the neutron fluence reached the value of $10^{12} \mathrm{n}$ $\mathrm{cm}^{-2}$, followed by even more pronounced changes in the sample irradiated with the fluence of $10^{13} \mathrm{n} \mathrm{cm}^{-2}$. The quality of SBDs was not satisfactory for DLTS measurements above 
the neutron fluence of $10^{14} \mathrm{n} \mathrm{cm}^{-2}$. Temperature dependent $\mathrm{C}-\mathrm{V}$ measurements revealed uniform changes in the free carrier concentration, showing a small decrease for the highest fluences.

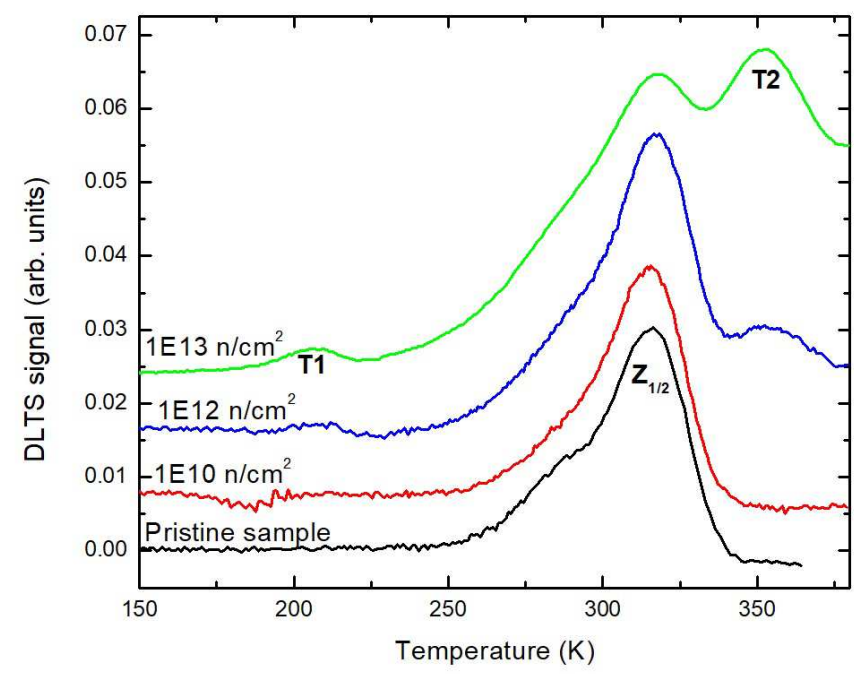

Fig. 2. DLTS spectra of pristine and selected neutron irradiated SBDs. Measurement setting are: emission rate $50 \mathrm{~s}^{1}$, reverse bias $V_{R}=10 \mathrm{~V}$, pulse bias $V_{P}=0.1 \mathrm{~V}$, and pulse width $\tau_{p}=10 \mathrm{~ms}$. The spectra are shifted vertically for clarity.

Fig. 2 shows DLTS spectra for the pristine SBD and selected neutron irradiated SBDs. In the pristine sample, only one broad and asymmetric DLTS peak is observed. The estimated activation energy for electron emission was 0.64 $\mathrm{eV}$. This defect, known as $Z_{1 / 2}$ has already been reported and was ascribed to the carbon vacancy $V_{C}(=/ 0)$ transition [14]. $V_{C}$ is known as one of the most stable defects in $\mathrm{SiC}$, acting as a strong minority carrier recombination center in n-type material. The intensity of $Z_{1 / 2}$ slightly increases with the neutron fluence. Additional DLTS peaks, which we have labelled as $\mathrm{T} 1$ and $\mathrm{T} 2$, have been detected for neutron fluences of $10^{12} \mathrm{n} \mathrm{cm}^{-2}$ and $10^{13} \mathrm{n} \mathrm{cm}^{-2}$, respectively. The activation energies for electron emission are estimated at 0.36 $\mathrm{eV}$ and $0.70 \mathrm{eV}$. The $\mathrm{T} 1$ and $\mathrm{T} 2$ traps have been reported in the literature in numerous instances, however, information regarding these defects, which regularly appear in implanted or irradiated $4 \mathrm{H}-\mathrm{SiC}$ samples are very limited and controversial. We suggest that $\mathrm{T} 1$ and $\mathrm{T} 2$ are intrinsic, simple radiation induced point-like defects.

\section{Prototype Detection System AND Initial TESTING}

Two main components of any detector system are 1) the detector of particle events and 2) the electronic system for processing and recording of events. The prototype detectors were based on hexagonal 4H-SiC-based Schottky diodes with several active layer thicknesses, ranging from $25 \mu \mathrm{m}$ to 170 $\mu \mathrm{m}$, their lateral dimensions being $1 \mathrm{~mm}$ by $1 \mathrm{~mm}$. The detectors consisted of SBDs surface-mounted onto chip carriers with electrical contacts. The chip carriers were enclosed in 3D-printed plastic holders in order to completely enclose the electrical contacts under high voltage (Fig. 3a). The holders had an opening above the SBD to enable charged particles to reach the SBD surface. The electronic system for particle event processing and recoding consisted of a preamplifier and a shaping amplifier PCB modules (CR-150-R5 and CR-160-R7) manufactured by CREMAT, and a digital signal processing multichannel analyser (DSP-MCA) manufactured by AMPTEK (model no. 8000D), connected to a laptop computer (Fig. 3b). Power to the electronic system (preamplifier and shaping amplifier) was provided by a standalone batterypowered voltage source, in order to avoid the use of mains power. The latter often carries electronic noise which can negatively affect the measurement performance. The voltage source also provided power to a separate high voltage bias module. Reverse negative bias was connected to the front Schottky contact, and the back Ohmic contact of the prepared prototype detectors was grounded. All initial tests have been performed in low-AC-noise environment of the ANSTO detector lab.

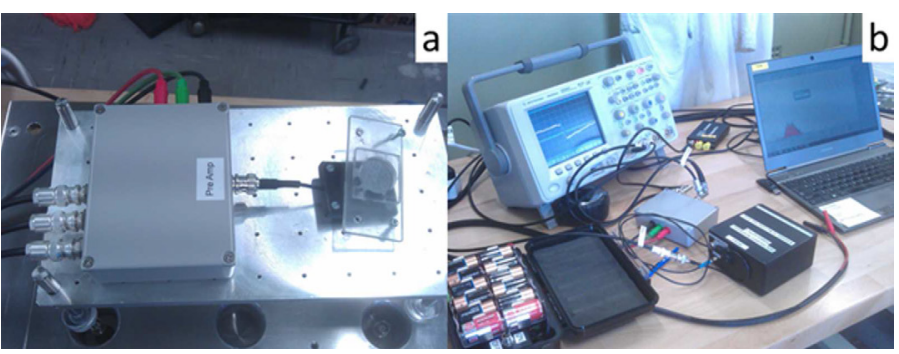

Fig. 3. Detector system under development: a) A SBD detector being tested using a ${ }^{241}$ Am source. b) Electronic system for operation of a detector and data acquisition during bench testing at ANSTO.

The detection set-up was tested using a precision pulse generator and a ${ }^{241} \mathrm{Am}$ source of energetic alpha particles. For a low capacitance input $(\sim 10 \mathrm{pF})$ to the preamplifier stage we used a CREMAT CR-110-R2 preamplifier chip and tested the detection energy resolution with the shaping PCB module equipped with the CR-200-R2 chip with shaping times of 0.5 and $1.0 \mu \mathrm{s}$. The total gain for event signal amplification was kept constant. Pole/Zero (P/Z) was adjusted for different shaping constants. Recorded events were sorted by the MCA in $2 \mathrm{k}$-channel energy spectra. The pulser peak and the ${ }^{241} \mathrm{Am}$ peak were fitted with Gaussians, given by the following expression:

$$
y=y_{0}+\frac{A}{w \sqrt{\frac{\pi}{4 \ln 2}}} \exp \left(-\frac{4 \ln 2\left(x-x_{c}\right)^{2}}{w^{2}}\right)
$$

where $y$ is the number of detected counts in the channel $x, x_{c}$ is the centroid, A determines the height of the peak and $w$ the peak width. Fig. 4 shows recorded alpha particle spectra. Although the energy resolution for line-pulse detection is very similar for reverse bias voltages of $50 \mathrm{~V}$ and $100 \mathrm{~V}$, the calculated width, FWHM and corresponding energy resolution for reverse bias of $50 \mathrm{~V}$ have lower values compared to reverse 
bias of $100 \mathrm{~V}$. Therefore we performed neutron detection tests using a reverse bias of $50 \mathrm{~V}$ and a shaping constant of $1 \mu \mathrm{s}$. The initial testing results demonstrated well the functionality of the detector prototypes and the electronic measurement system.

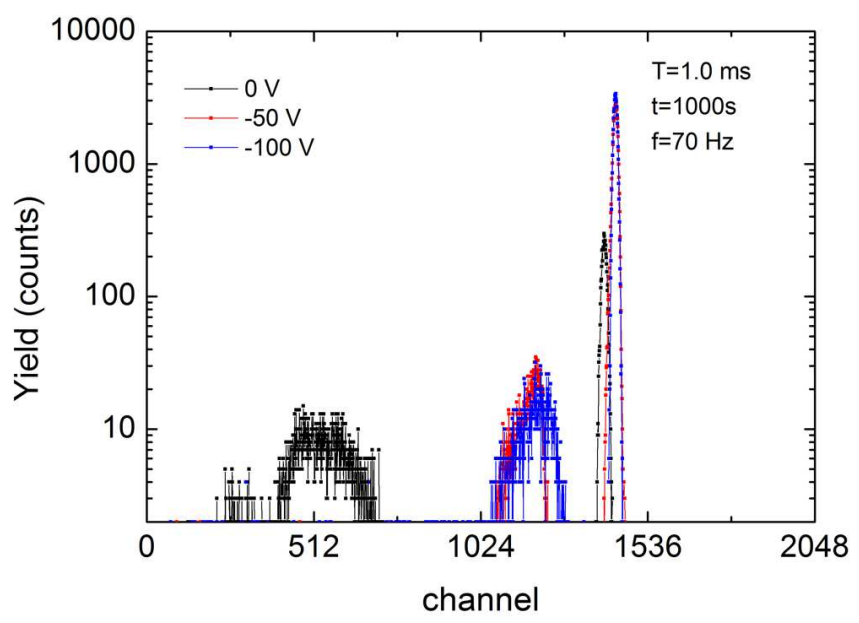

Fig. 4. Recorded ${ }^{241} \mathrm{Am}$ alpha particle spectra during initial testing with the prototype detector system and the following settings: precision pulse generator frequency: $70 \mathrm{~Hz}$, reverse bias voltages: $0 \mathrm{~V}$ (black), $-50 \mathrm{~V}$ (red) and -100 V (blue), total acquisition time: 1000s.

\section{TEST AND THE JSI TRIGA REACTOR}

Following the results obtained from the initial testing, an experimental campaign was performed at the JSI TRIGA reactor in May 2018 aiming at demonstrating the neutron detection capabilities of the prototype detectors. For the test, four new detectors were realized, enclosed in 3D-printed plastic holders, and housed in turn in custom-made aluminium enclosures. The detector prototypes were equipped with thermal neutron converting materials for neutron detection. Due to the very large thermal neutron cross sections of ${ }^{10} \mathrm{~B}$ and ${ }^{6} \mathrm{Li}$ isotopes (around $3843 \mathrm{~b}$ and $938 \mathrm{~b}$ respectively, at an incident neutron energy of $0.0253 \mathrm{eV}$ [15]), the chosen converting materials were enriched ${ }^{10} \mathrm{~B},{ }^{6} \mathrm{LiF}$ and ${ }^{10} \mathrm{~B}_{4} \mathrm{C}$ powders. The ${ }^{10} \mathrm{~B}$ and 6

LiF powders were applied onto plastic film and mounted above the openings of the 3D-printed SBD holders. One of the SBDs was realized with a capping layer of ${ }^{10} \mathrm{~B}_{4} \mathrm{C}$, in physical contact with the SBD. For the preparation of the ${ }^{10} \mathrm{~B}_{4} \mathrm{C}$ capping layer, firstly, the whole surface of a SiC SBD mounted onto a chip carrier was covered with an adhesive material in order to prevent leakage current. After drying, ${ }^{10} \mathrm{~B}_{4} \mathrm{C}$ powder was dropped onto the adhesive surface on the SiC SBD and encased in a second adhesive layer. Fig. 5 shows two realized prototype detectors used in the experimental tests (fully enclosed and open).

Firstly, the detectors were tested with no neutron converters (except for the detector with the ${ }^{10} \mathrm{~B}_{4} \mathrm{C}$ capping layer) and no radiation sources. All the obtained spectra showed a large peak at low channel numbers, which was attributed to electronic noise, and no counts appeared at higher channels. It was also

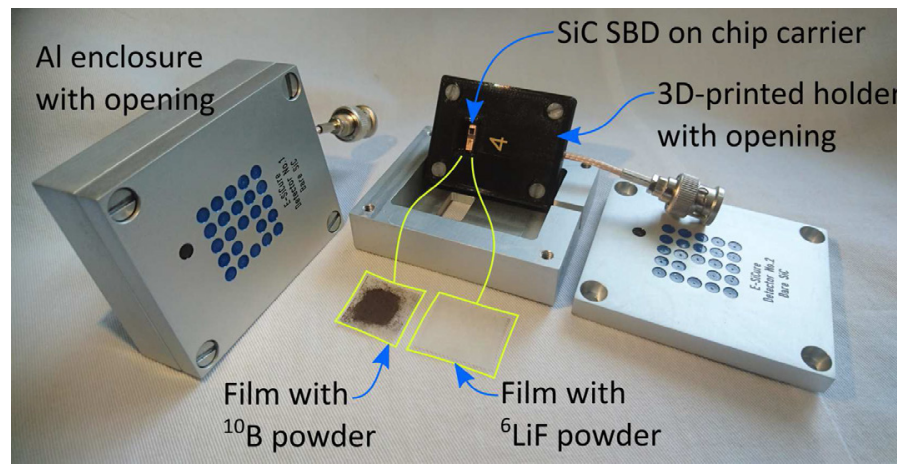

Fig. 5. E-SiCure prototype detectors. Left: assembled detector prototype in aluminium enclosure. Right: prototype detector components: SiC SBD mounted onto chip carrier with contacts, installed in $3 \mathrm{D}$ printed holder with opening, converter films (with ${ }^{10} \mathrm{~B}$ and ${ }^{6} \mathrm{LiF}$ powder), open aluminium enclosure with opening.

observed that counts started to appear at higher channels if the laptop computer was connected to mains power or if the mousepad of the laptop computer was touched. Therefore, all the subsequent measurements were performed with the laptop computer running only on battery power and using a wireless mouse for control. The detectors were then tested using a ${ }^{241} \mathrm{Am}$ source, its nominal activity being $416 \mathrm{kBq}$ (reference date 29.5.2018). For the three detectors without the ${ }^{10} \mathrm{~B}_{4} \mathrm{C}$ capping layer a clear peak due to the alpha particles emitted from ${ }^{241} \mathrm{Am}$ at high channels was observed, confirming the functioning of the SBDs as detectors of charged particles. The peak was not observed for the detector with the ${ }^{10} \mathrm{~B}_{4} \mathrm{C}$ capping layer, indicating that the detector was not functioning as expected. Following the functioning tests, the neutron converter films were applied to the detectors and neutron irradiations were performed in the Dry Chamber of the JSI TRIGA reactor. The Dry Chamber is a large irradiation room in the concrete body of the reactor, connected with the reactor core by a graphite thermalizing column [16]. It is mostly used for radiation hardness testing of detectors, electronic components and systems [17][18]. Measurements were taken firstly with a $25 \mu \mathrm{m}$ thick prototype detector equipped with ${ }^{10} \mathrm{~B}$ converter film at the following power levels: $10 \mathrm{~kW}, 50 \mathrm{~kW}, 100 \mathrm{~kW}, 180 \mathrm{~kW}$ and $250 \mathrm{~kW}$. In all the recorded spectra we observed a significant number of counts at higher energy channels, attributed to alpha particles and recoil ${ }^{7} \mathrm{Li}$ particles originating from ${ }^{10} \mathrm{~B}(n, \alpha)$ reactions. We also observed a distinctive structure in the spectra, attributed to the different energies of the secondary particles. Fig. 6 shows the recorded spectra using the ${ }^{10} \mathrm{~B}$ converter film.

Measurements were continued with a $69 \mu \mathrm{m}$ thick prototype detector equipped with ${ }^{6} \mathrm{LiF}$ converter film, at the following reactor power levels: $10 \mathrm{~kW}, 50 \mathrm{~kW}, 100 \mathrm{~kW}, 170 \mathrm{~kW}$ and $250 \mathrm{~kW}$. As for the ${ }^{10} \mathrm{~B}$ converter film, with the ${ }^{6} \mathrm{LiF}$ converter film we also observed a significant number of counts at higher energy channels, attributed to tritons and alpha particles originating from ${ }^{6} \mathrm{Li}(n, t)$ reactions. We also observed a distinctive structure in the spectra, which was qualitatively different from 


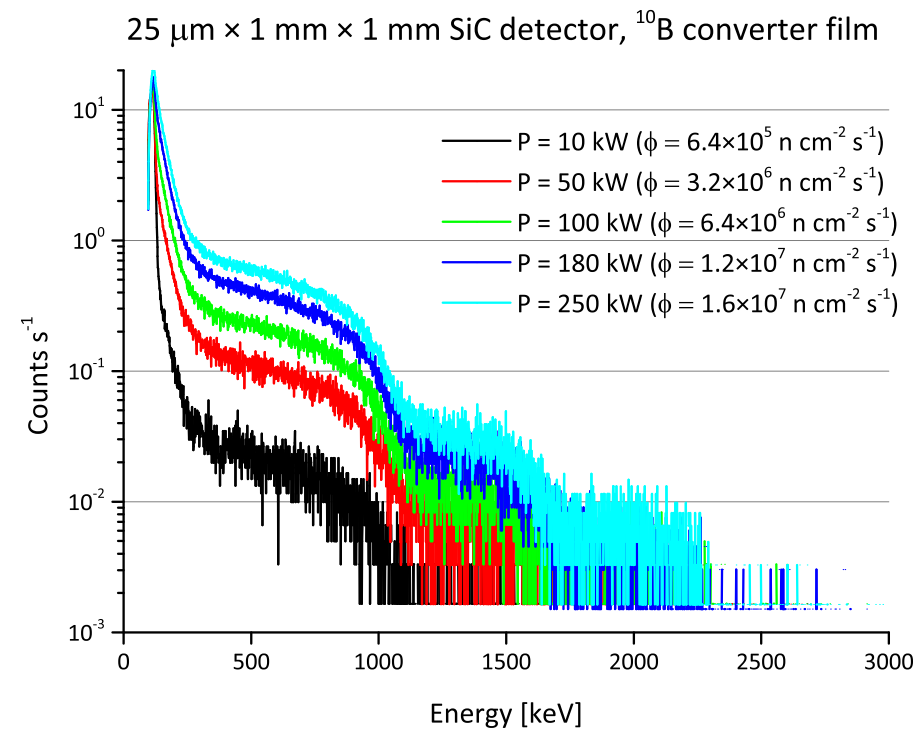

Fig. 6. Measured spectra with a $25 \mu \mathrm{m} \mathrm{SiC}$ detector equipped with ${ }^{10} \mathrm{~B}$ converter film in the JSI TRIGA reactor Dry Chamber at different reactor power levels.

the one observed using ${ }^{10} \mathrm{~B}$ converter film. Fig. 7 shows the recorded spectra using the ${ }^{6} \mathrm{LiF}$ converter film.

\section{$69 \mu \mathrm{m} \times 1 \mathrm{~mm} \times 1 \mathrm{~mm}$ SiC detector, ${ }^{6} \mathrm{LiF}$ converter film}

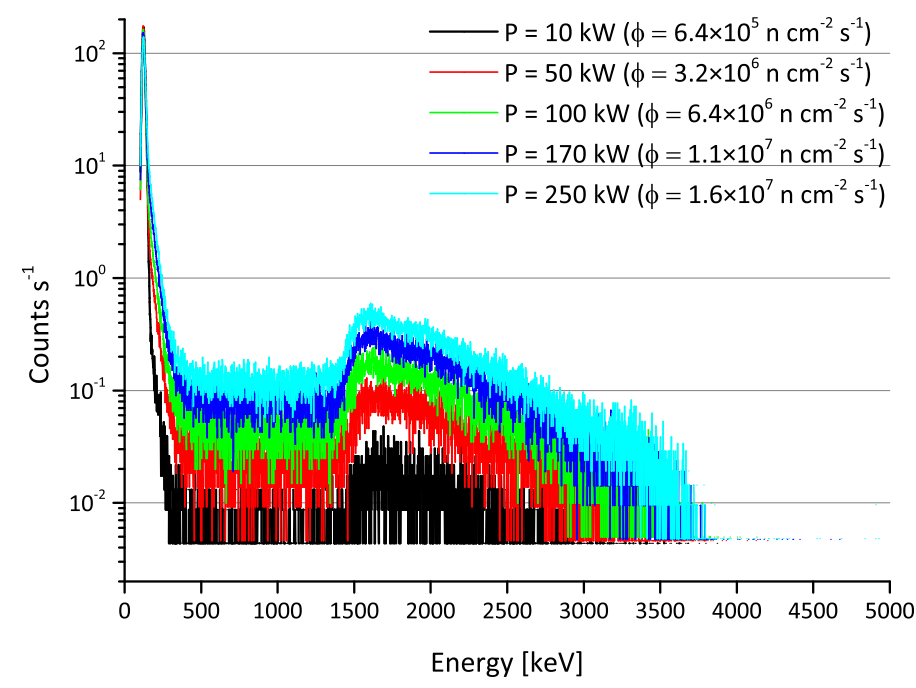

Fig. 7. Measured spectra with a $25 \mu \mathrm{m} \mathrm{SiC}$ detector equipped with ${ }^{6} \mathrm{LiF}$ converter film in the JSI TRIGA reactor Dry Chamber at different reactor power levels.

\section{CONCLUSIONS AND Future WORK}

This paper gives an overview of the activities performed at the JSI TRIGA reactor in the framework of the E-SiCure project. The project aims at studying $\mathrm{SiC}$ material properties for the detection of SNM for border and port security. SiCbased SBDs displayed very good radiation hardness properties. Irradiation tests of detector prototypes, equipped with ${ }^{10} \mathrm{~B}$ and ${ }^{6} \mathrm{LiF}$ converter films were performed in the Dry Chamber of the reactor, clearly demonstrating the ability of small $\mathrm{SiC}$ SBDs (side dimensions of $1 \mathrm{~mm} \times 1 \mathrm{~mm}$, thickness below $200 \mu \mathrm{m}$ ) to detect alpha and recoil charged particles originating from neutron interactions. Further studies and irradiation testing will be carried within the E-SiCure project with the objective to optimize the detector and converter properties for maximal neutron detection efficiency.

\section{ACKNOWLEDGMENT}

The present work was financially supported by the NATO Science for Peace and Security Programme, under project no. SPS 985215 - Engineering Silicon Carbide for Border and Port Security E-SiCure. The JSI project team would like to acknowledge the support from the Slovenian Ministry of Education, Science and Sport (project no. P2-0073 - Reactor Physics). The RBI project team would like to acknowledge financial support from the European Regional Development Fund for the Center of Excellence for Advanced Materials and Sensing Devices (Grant No. KK.01.1.1.01.0001). JC acknowledges the Fundação para a Ciência e a Tecnologia (FCT) through project UID/CTM/50025/2013.

\section{REFERENCES}

[1] M. Ito, L. Storasta, H. Tsuchida, "Development of 4HSiC Epitaxial Growth Technique Achieving High Growth Rate and Large-Area Uniformity" Appl. Phys. Expr. 1 (2008) 015001.

[2] J.T. Goorley et al., 'Initial MCNP6 Release Overview - MCNP6 version 1.0", LA-UR-13-22934 (2013).

[3] M.B. Chadwick, et al., ENDF/B-VII.1 nuclear data for science and technology: cross sections, covariances, fission product yields and decay data, Nucl. Data Sheets, 112, 2011, pp. 2887-2996.

[4] R. Jeraj, M. Ravnik, TRIGA mark II reactor: U(20)-zirconium hydride fuel rods in water with graphite reflector, IEU-COMP-THERM-003, International Handbook of Evaluated Criticality Safety Benchmark Experiments, NEA/NSC/DOC(95)03, OECD-NEA, Paris, France, 2010.

[5] International Handbook of Evaluated Critical Safety Benchmark Experiments, Organization for Economic Cooperation and Development, Nuclear Energy Agency, NEA/NSC/DOC(95)03, Paris (published on DVD).

[6] A. Kolšek et al., Using TRIGA Mark II research reactor for irradiation with thermal neutrons, Nucl. Eng. Des, 283, 2015, pp. 155-161.

[7] V. Radulović et al, Qualification of heavy water based irradiation device in the JSI TRIGA reactor for irradiations of FT-TIMS samples for nuclear safeguards, Nucl. Inst. Meth. A, 885, 2018, pp. 139-144.

[8] V. Radulović et al, Report on experimental tests of a combined SPND and thermocouple sensor for the DISCOMS project at the JSI TRIGA reactor, JSI report No. 12547, Joef Stefan Institute, 2018.

[9] L. Snoj et al., Calculation of kinetic parameters for mixed TRIGA cores with Monte Carlo, Ann. Nucl. Energy, 37 (2), 2010, pp. 223-229.

[10] L. Snoj et al., Analysis of neutron flux distribution for the validation of computational methods for the optimization of research reactor utilization, Appl. Radiat. Isot., 69 (1), 2011, pp. 136-141.

[11] V. Radulović et al., Validation of absolute axial neutron flux distribution calcula- tions with MCNP with ${ }^{197} \mathrm{Au}(n, \gamma){ }^{198} \mathrm{Au}$ reaction rate distribution measurements at the JSI TRIGA Mark II reactor, Appl. Rad. Isot., 84, 2014, pp. 57-65.

[12] L. Snoj, G. Žerovnik, A. Trkov, Computational analysis of irradiation facilities at the JSI TRIGA reactor, Appl. Rad. Isot., 70 (3), 2012, pp. 483-488.

[13] A. Trkov, V. Radulović, L. Snoj, The GRUPINT neutron spectrum adjustment code - general features and characterization of the spectra in three irradiation channels of the JSI TRIGA reactor, Program and Book of abstracts, 16th International Symposium on Reactor Dosimetry, May 7 - 12, 2017, Santa Fe, USA.

[14] I. Capan et al., Double negatively charged carbon vacancy at the h- and k-sites in 4H-SiC: combined Laplace-DLTS and DFT study. Journal of applied physics, 123 (16), 2018, pp. 161597-1-161597-6. 
[15] R. Capote et al., "Updating and Extending the IRDF-2002 Dosimetry Library," Journal of ASTM International, 9 (4), 2012, pp. 1-9.

[16] V. Radulović et al., Characterization of ex-core irradiation facilities of the JSI TRIGA Mark II reactor, Proceedings, 21st International Conference Nuclear Energy for New Europe, September 5-7, 2012, Ljubljana, Slovenia.

[17] I. Mandić et al., "Bulk damage in DMILL npn bipolar transistors caused by thermal neutrons versus protons and fast neutrons", IEEE Transactions on Nuclear Science, 51 (4), 2004, pp. 1752-1758.

[18] I. Mandić, V. Cindro, A. Gorišek, G. Kramberger and M. Mikuž, "Online Integrating Radiation Monitoring System for the ATLAS Detector at the Large Hadron Collider", IEEE Transactions on Nuclear Science, 54 (4), 2007, pp. 1143-1150. 